\title{
Improving communications between theoretical ecologists, mathematical ecologists, and ecological modelers: response to the critique of our book How species interact
}

\author{
Roger Arditi • Lev R. Ginzburg
}

Received: 13 September 2013 / Accepted: 8 October 2013 / Published online: 24 October 2013

(C) Springer Science+Business Media Dordrecht 2013

Keywords Trophic interactions · Prey dependence · Predator interference $\cdot$ Ratio dependence $\cdot$ Ecological theory

We thank the editor in chief, Alan Hastings, for giving us the opportunity to publish this response to the critical paper in this issue (Barraquand 2013). Since we were asked to write a short response, we will focus only on the author's main point. The critique addresses many details of our book (Arditi and Ginzburg 2012), but responding to all of it would require repeating much of the book because it already contains most answers. The controversy about ratio dependence was substantially settled with the joint paper of Abrams and Ginzburg (2000). So, focusing only on the author's main point will lead us to an explanation of how this critique originates and will allow us to address a more general issue that may be of interest to the readers of Theoretical Ecology.

The main argument constructed by Frédéric Barraquand (FB) is that the prey-dependent model is a "straw man" artificially erected by us in order to cut it down in favor of the opposing "limit myth" of ratio dependence. FB's position is that there are many other models besides these two classes, and all have their places under the sun; different models are needed for different purposes. The latter statement is so general that it is bound to be correct. We never said that the

R. Arditi

Ecologie et Evolution, Université Pierre et Marie Curie, Sorbonne Universités, Case 237, 75231 Paris Cedex 05, France

R. Arditi $(\bowtie)$

Ecology and Evolution, University of Fribourg, Chemin du Musée 10, 1700 Fribourg, Switzerland

e-mail: roger.arditi@unifr.ch

L. R. Ginzburg

Ecology and Evolution, Stony Brook University, Stony Brook, NY 11794, USA ratio-dependent model would give an exact description of every existing trophic community. What we say is that it is a good "null model," i.e., a good starting point. All ecologists, even the most empirical, have some theory in their minds, and what ecologists view as the "default theory" can serve as another definition of the null model. Our meaning in this regard was clear, as shown by the various reviewers who understood fully our book's purpose (DeAngelis 2013; Fryxell 2013; Krebs 2013; Peterson 2013). (We note in passing that, while FB uses some of Krebs's work on cycles in support of his critique, Charles Krebs actually wrote a very favorable review of our book.)

As we explained in our book, our theory can be traced back to a number of precursors; we mentioned Kolmogorov, Leslie (in part only), and Contois, and we recently found out that Ivlev too had been led to the same idea in his work with fish (Ivlev 1961). Both Contois and Ivlev were experimentalists and developed ratio-dependent models on empirical grounds. Although FB calls for pragmatism, he hardly mentions the many direct tests of functional responses against data that we present in our book, chapter 2. There, we show that most experimental estimates of interference fall on a spectrum of intermediate values, but closer to the ratio-dependent end than to the prey-dependent. If we simplify by choosing the ratiodependent end, not only do we gain in parsimony (admittedly, a little), but we obtain a very satisfactory model on theoretical grounds because of its symmetry (see below).

FB's proposition to use various models to explain a variety of trophic relations is unsatisfactory. Given that our theory, which he criticizes, unifies the explanation of all of these phenomena, we see no compelling reason for preferring a multiplicity of approaches. Moreover, beyond criticism of our theory, FB targets the whole theoretical school that uses deterministic models. He suggests instead stochastic population models and time series analysis. We have nothing against log-linear Gompertz-like predator-prey stochastic 
models. The linear approximation in a $\log$ scale is very useful for data analysis (when data are available). This, in our view, is a matter totally unrelated to our book since log-linear models will approximately work on the basis of any of the models. Similarly, adding complex mathematical details can be justified in order to describe specific biological cases for which available data are abundant. Such elaborate ecological modeling is necessary but, again, unrelated to the aims of our book.

Not everyone would like to accept it, but as FB's paper illustrates, there are noticeable differences between theoretical ecology, mathematical ecology, and ecological modeling. Mathematicians export equations from theoretical ecology and do mathematical and/or statistical work based on them. This is not different from the distinction between mathematical physics and theoretical physics. Modelers too, not unlike engineers using physics, export basic models from theoretical ecology and complexify them in order to better describe specific systems. Theoretical ecologists worry about basic models and their founding principles and logical structure, in an effort to identify general properties. For instance, the conversion principle is a part of theoretical ecology and so is the critique of Leslie's violation of it (Maynard Smith 1974, our book section 1.7).

It is instructive to note that $\mathrm{FB}$, while doing a vigilant job of criticizing nearly all of our book chapters, did not touch the main point of chapter 6 , which is entitled "It must be beautiful." In it, we show why the ratio-dependent model is the only one that is consistent with the axiom of single species exponential growth. This, of course, does not prove us right. Malthusian growth has its own assumptions, and if it is not accepted as a model of "undisturbed dynamics," our argument will fail too, but if we keep the exponential growth at the basis of theoretical ecology, there are good reasons to accept ratiodependent predation as basic too, in order to coordinate assumptions at the foundations.

While the distinctions in the use of models in ecology were already discussed in the 1960s (e.g., Levins 1966), there is a resurgence of this debate, with a recent paper (Evans et al. 2013) challenging Robert May's well-known call for simple models that "sacrifice precision in an effort to grasp at general principles" (May 1973). In his opening editorial to the first issue of this journal, Theoretical Ecology, Alan Hastings wrote that "almost all of the influential studies have in common simplicity, elegance, and generality" (Hastings 2008). While we strive to work along these lines, we also think that all subfields, theoretical ecology, mathematical ecology, and ecological modeling contribute to the joint enterprise of moving the vast field of ecology forward in their own ways. We hope that this journal will contribute to debates that will lead to more mutual understanding between the various schools.

\section{References}

Abrams PA, Ginzburg LR (2000) The nature of predation: preydependent, ratio-dependent, or neither? Trends Ecol Evol 15:337341

Arditi R, Ginzburg LR (2012) How species interact: altering the standard view on trophic ecology. Oxford University Press, Oxford, $170 \mathrm{p}$

Barraquand F (2013) Functional responses and predator-prey models: a critique of ratio dependence. Theor Ecol. doi:10.1007/s12080-0130201-9

DeAngelis DL (2013) The case for ratio dependence in trophic ecology. Trends Ecol Evol 28:259-260

Evans MR et al (2013) Do simple models lead to generality in ecology? Trends Ecol Evol 28:578-583

Fryxell J (2013) The great predator-prey debate. Ecology 94:1206-1207

Hastings A (2008) Editorial - an ecological theory journal at last. Theor Ecol 1:1-4

Ivlev VS (1961) Experimental ecology of the feeding of fishes. Yale University Press, San Diego, p 302, Translation of original book published in Russian in 1955

Krebs CJ (2013) Review of the book How species interact. Q Rev Biol $88: 37$

Levins R (1966) The strategy of model building in population biology. Am Sci 54:421-431

May RM (1973) Stability and complexity in model ecosystems. Princeton University Press, Princeton

Maynard Smith J (1974) Models in ecology. Cambridge University Press, Cambridge, $146 \mathrm{p}$

Peterson RO (2013) It's a wonderful gift. Science 339:142-143 